\title{
Crashworthiness Optimization of Thin-Walled Rail with Different Collision Boundary Conditions
}

\author{
Hequan $\mathrm{Wu}^{*}{ }^{* 1,2}$ Libo $\mathrm{Cao}^{1}$ Hongfeng $\mathrm{Mao}^{2}$ \\ ${ }^{I}$ State Key Laboratory of Advanced Design and Manufacturing for Vehicle Body, Hunan University, Changsha, 410082, \\ P.R. China \\ ${ }^{2}$ Key Laboratory of Lightweight and Reliability Technology for Engineering Vehicle, College of Hunan province, \\ Changsha University of Science and Technology, Changsha, 410114, P.R. China
}

\begin{abstract}
As the world automotive crash safety regulations are different, it's very important to design the energy absorbing structures that satisfy different collision boundary conditions. A large number of vehicle energy absorption beams dimensions were measured and then a common thin-walled rail was chosen. Considering the complexity of automobile collision boundary, finite element analysis and experimental design, interval uncertain algorithms, Kriging approximate model, NSGA - II genetic algorithm were combined to optimize the structure of the thin-walled rail with different impact velocity and different impact angle. Then the Pareto optimal solution was obtained. Thin walled beam after optimization has good energy absorption characteristics under different collision boundary conditions. Research results provide a method for the designing of a car that meets various crash regulations at the same time.
\end{abstract}

Keywords: Boundary conditions, crashworthiness, optimal design, thin-walled rail.

\section{INTRODUCTION}

The thin-walled rails are the main components of the automobile bearing structure. Their quality is small, while the axial strength is higher. The thin-walled rails can provide stable axial compression force and they have good energy absorption characteristics. These rails play the most important role in absorbing crush energy during vehicle collision. Many experimental and theoretical studies have been carried out on plastic collapse of thin-walled rails of various sizes and materials by the domestic and foreign scholars [1]. However, these studies are mostly based on a specific impact of regulations. The lack of uniform international standards, there is a difference in the world automobile crash regulations.

For example, In China, Regulation CMVR294 points out that $48 \mathrm{~km} / \mathrm{h}$ is the velocity of front crash and Regulation CNCAP stipulates $50 \mathrm{~km} / \mathrm{h}$ is the front crash velocity and $64 \mathrm{~km} / \mathrm{h}$ is the $40 \%$ off-set collision velocity, whereas in Europe, $40 \%$ offsetting velocities are legitimated $56 \mathrm{~km} / \mathrm{h}$ in ECE94 and $64 \mathrm{~km} / \mathrm{h}$ in Euro-NCAP. In the US, the velocity in vehicle front collision is at $48.3 \mathrm{~km} / \mathrm{h}$. In the fixed rigid barrier crash test, collision happened in a 30 degrees range both left and right sides at the front of the automobile should meet the requirements of occupant protection as the FMVSS208 regulated.

Admittedly, the crash velocity and angle regulated by different countries fluctuate within a certain range. Plus, uncertainties remain in collision boundary conditions of specific traffic accidents in reality (such as the crash velocity and angle). To design the thin-walled rail energy absorption structure that enables vehicles to possess rather stable characteristics of energy absorption with any possible collision boundary conditions is the key in this paper.

\section{PROBLEM DESCRIPTIONS}

Sizes of energy absorption thin-walled rail for automobile are dispersed by particular vehicle types. The section shape of selected thin-walled rail from massive measurements of authentic vehicle types' energy absorption beam sizes, proposed in this project, is depicted as shown in Fig. (1).

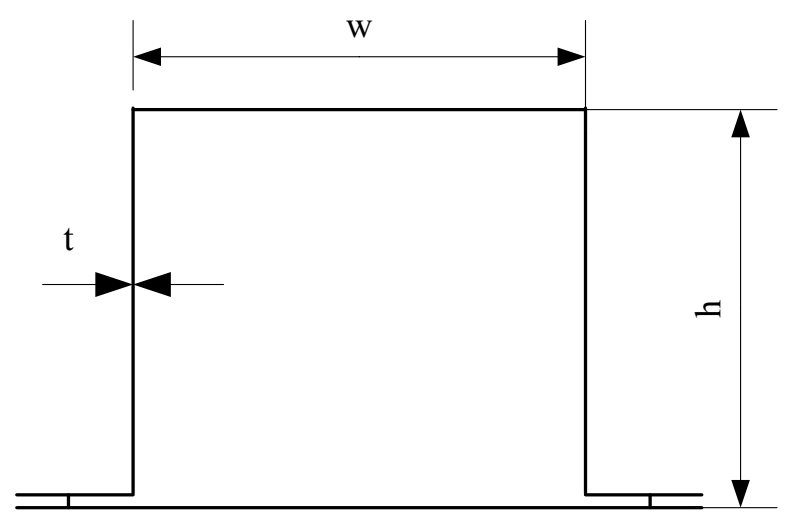

Fig. (1). The section shape of thin-walled rail.

The main parameters contributing to characteristics of thin-walled rail energy absorption include section width $w$, 
section height $h$ and section thickness $t$, in accord with literatures [2] used in the paper. Define $X=(w, h, t)^{T}$ as design variable.

Consider vehicle safety regulations are mainly countries in the world, combined with the actual situation of road traffic accidents, the impact velocity and impact angle range of thin-walled rails selected in this paper is that:

Taking automotive crash safety regulations and the actual situation of road traffic accidents into consideration, the impact velocity $v$ and collision angle $\alpha$ range of thin-walled rails selected in this paper is that: $v \in(30,50), \alpha \in(0,30)$. Define $U=(v, \alpha)^{T}$ as interval variable.

The passive safety of vehicles is proportional to the amount of energy that thin-walled rail consumers. The more thin-walled rail absorb energy, the less energy and harm may transmit to passenger compartment. Energy absorbed during the collision (the total strain energy) consists of both elastic strain energy and plastic strain energy, shorted for $E$.

On the other hand, the average impulsive load $F$ during the impact is also considered as one of the critical design objectives to prevent the occupant's body from severe biomechanical injury, inasmuch as the load should be as small as possible.

Besides, due to the limited space of energy absorbing when vehicle collision, if excessive deformation of the collision site, it may cause occupant injury. For example, in a frontal collision, excessive deformation is likely to penetrate the engine compartment and the cab drivers, which lead to occupant injury. Hence, it's necessary to limit the deformation distance of energy absorption segments

The main objectives of crashworthiness safety design optimization are as follows: Maximize total energy absorption and minimize average impulsive load as long as maximum deformation amount meets requires. Its optimization model can be written as:

$$
\begin{array}{ll}
\text { Min } & -E(X, U) \\
& F(X, U) \\
\text { s.t. } & g_{\min } \leq g(X, U) \leq g_{\max } \\
& X^{L} \leq X \leq X^{U}
\end{array}
$$

where, $g_{\min }, g_{\max }$ stand for upper and lower limits of allowable deformation amount; $X^{L}, X^{U}$ stand for upper and lower limits of design variables

The range of the design variables is obtained by using the measurement on the vehicle thin-walled rail's size. It is as follows: $1 \leq t \leq 4,60 \leq w \leq 120,60 \leq h \leq 120$. Considering the limitation of engine compartment at the front part of vehicles, the deformation amount of thin-walled rail is defined as follows: $g_{\min }=300, g_{\max }=400$.

\section{VERIFICATION OF FINITE ELEMENT MODEL}

Build CAD model of the thin-walled rail, transfer it into IGES format, and then import it into HYPERMESH software.
Three-node and four-node shell elements are used to mesh in the finite element model. The basic size of the shell element is chosen as $10 \mathrm{~mm}$. The impact model is erected as shown in Fig. (2).

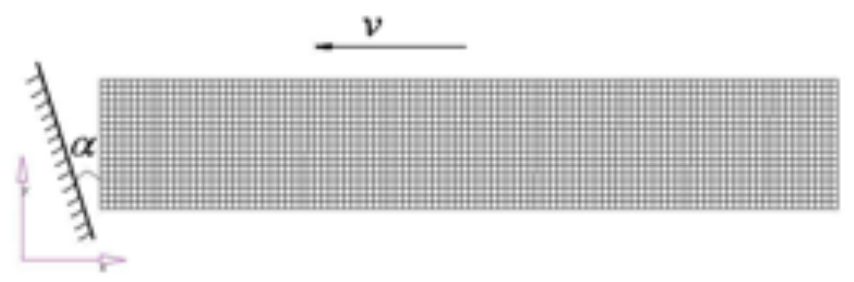

Fig. (2). Finite element model of thin-walled rail's impact.

The length of thin-walled rail is set as $500 \mathrm{~mm} .500 \mathrm{~kg}$ concentrated nodal-mass is added to thin-walled rail's free end. Thin-walled rail has a movement to the negative direction of axis $x$ at an initial speed of $v$ and $\alpha$ which is the angle between rigid wall and axis $y$.

The nonlinear FE simulations were conducted using the explicit code PAM-CRASH. Low carbon steel material is applied to thin-walled rail. The elasticity modulus of the material is $2.1 \times 10^{6} \mathrm{MPa}$, Poisson's ratio is 0.3 and the density is $7.8 \times 10^{3} \mathrm{~kg} . \mathrm{m}^{-3}$. A single surface contact algorithm was used in the simulation. During the simulations, the thinwalled rail was restricted to move and deform only in its main plane, i.e. The FE simulations were conducted for $40 \mathrm{~ms}$.

To verify the effectiveness of finite element mode, sled test must be carried out. The test at a collision velocity of $30 \mathrm{~km} / \mathrm{h}$ and a 0 degree angle in the sled test located at State Key Laboratory of Advanced Design and Manufacturing for Vehicle Body of Hunan University. The thin-walled rail welded to the front of sled, which has the same section size as shown in Fig. (1). Two acceleration sensors are erected at mass center of left and right ventricular long axes. The initial velocity and overall quality of the simulation model are consistent with the sled.

As can be seen from Fig. (3), the deformed plot of the finite element simulation and sled test collision are basically the same, which verified the accuracy of the finite element model.

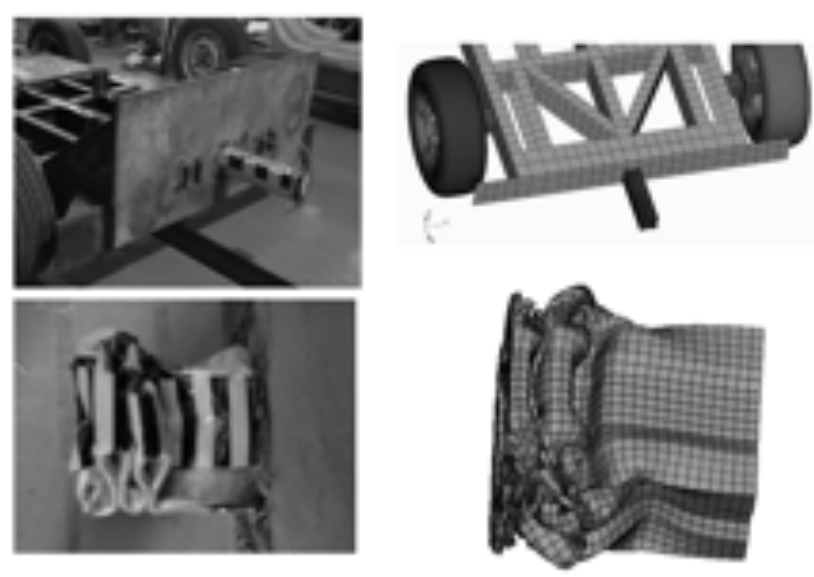

Fig. (3). Comparison of sled test and simulation. 


\section{OPTIMAL DESIGN METHODOLOGIES FOR UNCERTAINTY INTERVAL}

The crash velocity and angle are uncertain in traffic accidents and their value changes within a certain range. They are related to problems of interval number.

\subsection{Treatments to Objective Functions}

To interval numbers $\mathrm{A}$ and $\mathrm{B}$, orders of intervals manifest the superiority of one to another. Literature [3] provides a symbol " $\leq_{m w}$ " to rank the interval numbers. It can be seen that in the Eq. (3)

Literature [3] provides a definition to rank the interval numbers of minimization problem when defining the relationship of interval

$$
A \leq_{m w} B . \begin{cases}A^{I} \leq_{c w} B^{I} & \text { only when } A^{c} \geq B^{c} \text { and } A^{w} \geq B^{w} \\ A^{I}<_{c w} B^{I} & \text { only when } A^{I} \leq_{c w} B^{I} \text { and } A^{I} \neq B^{I}\end{cases}
$$

where:

$$
\begin{aligned}
& m(A)=\frac{A^{L}+A^{R}}{2}, w(A)=\frac{A^{R}-A^{L}}{2}, \\
& m(B)=\frac{B^{L}+B^{R}}{2}, w(B)=\frac{B^{R}-B^{L}}{2}
\end{aligned}
$$

In Eq. (3), $m$ stands for midpoint and $w$ stands for interval radium; $L$ and $R$ are superscripts stand for left and right boundary of interval.

Use interval order " $\leq_{m w}$ " to represent the objective function in Eq. (2). Midpoint and radium of objective functions intervals are expected to be the smallest. Then Eq. (2) can be converted into certainty objective problems as follows:

$$
\left\{\begin{array}{l}
\min _{\mathrm{X}}[m(-E(\mathrm{X}, \mathrm{U})), w(-E(\mathrm{X}, \mathrm{U}))] \\
\min _{\mathrm{X}}[m(F(\mathrm{X}, \mathrm{U})), w(F(\mathrm{X}, \mathrm{U}))]
\end{array}\right.
$$

where:

$$
\begin{gathered}
m(-E(\mathrm{X}, \mathrm{U}))=\frac{1}{2}\left(E^{L}(\mathrm{X})+E^{R}(\mathrm{X})\right), w(-E(\mathrm{X}, \mathrm{U}))=\frac{1}{2}\left(E^{R}(\mathrm{X})-E^{L}(\mathrm{X})\right) \\
m(F(\mathrm{X}, \mathrm{U}))=\frac{1}{2}\left(F^{L}(\mathrm{X})+F^{R}(\mathrm{X})\right), w(F(\mathrm{X}, \mathrm{U}))=\frac{1}{2}\left(F^{R}(\mathrm{X})-F^{L}(\mathrm{X})\right) \\
E^{L}(\mathrm{X})=\min _{U \in \Gamma} E(\mathrm{X}, \mathrm{U}), E^{R}(\mathrm{X})=\max _{U \in \Gamma} E(\mathrm{X}, \mathrm{U}), \\
F^{L}(\mathrm{X})=\min _{U \in \Gamma} F(\mathrm{X}, \mathrm{U}), F^{R}(\mathrm{X})=\max _{U \in \Gamma} F(\mathrm{X}, \mathrm{U}) \\
\Gamma=\{(30 \leq v \leq 50) \bigcup(0 \leq \alpha \leq 30)\}
\end{gathered}
$$

Uncertainty objective functions are changed into certainty objective functions. The weighted sum for all objectives achieves objective evaluation functions as follows:

$$
\left\{\begin{array}{l}
\min _{X}(-E(X, U))=(1-\beta) m(-E(X, U))+\beta w(-E(X, U)) \\
\min _{X}(F(X, U))=(1-\beta) m(F(X, U))+\beta w(F(X, U))
\end{array}\right.
$$

In Eq. (5), $\beta$ is the weight coefficient, $0 \leq \beta \leq 10.5$ is taken in the thesis.

\subsection{Treatments to Constraint Functions}

The constraint function $g(X, U)$ in Eq. (2) is also an uncertainty amount related to interval ranges, however it can be converted to certainty problem by introducing penalty function ${ }^{[5,6]}$ as follows:

$\sigma \cdot \varphi\left(P\left(C^{I} \geq D^{I}\right)-\lambda\right)$
$=\sigma \cdot\left(\max \left(0,-\left(P\left(C^{I} \geq D^{I}\right)-\lambda\right)\right)\right)^{2}$

where $\varphi$ stands for penalty function. A bigger value is taken for penalty factor $\sigma .1000$ is taken in the thesis.

Intervals: $C^{I}=\left[g^{L}(\mathrm{x}), g^{R}(\mathrm{x})\right], D^{I}=\left[g_{\text {min }}, g_{\max }\right] C^{I}$ is the interval value of uncertainty constraint function and $g^{L}(\mathrm{x})$, $g^{R}(\mathrm{x})$ are the upper and lower boundaries of constraint function; $D^{I}$ is the allowable constraint interval range; $P\left(C^{I} \geq D^{I}\right)$ means the probability that $C^{I}$ is bigger than $D^{I}$

; $\lambda$ is the given value of probability- 0.8 is given in the passage.

If the constraints to meet the requirements, the penalty function's value is 0 ; otherwise, penalty should be attached after objective function.

\section{OPTIMAL DESIGN METHODOLOGIES FOR NSGA-II}

Solutions for multi-objective optimization problem is usually a set, these solutions on all terms of the objective function can not compare their merits. These solutions called non-dominated solutions or called Pareto optimal solution. NSGA-II is one of the most typical arithmetics [4], as shown in Fig. (4). Firstly, the first filial generation population is harvested after the initial population of $\mathrm{N}$ reproduced randomly undergoes non-dominated sorting and selection, crossover, mutation of genetic algorithm. Sencondly, rapid non-dominated sorting initiates after filial generation population and initial population have been combined since the second generation. Crowding degree calculation is, at the same time, set out on individuals of every non-dominated layer that members of the ascertained new initial populations are suitable indivduals selected by non-dominated relation and crowding degree. Finally, it is not until the filial generation contends conditions to conclude the program will has the offspring stopped coming out and the like.

\section{OPTIMAL DESIGN FOR THIN-WALLED RAIL CRASHWORTHINESS}

The crashworthiness optimal design for thin-walled rail combines finite element analysis with experiment design, approximation model and NSGA-II algorithm. The flow chart was shown in Fig. (3) 


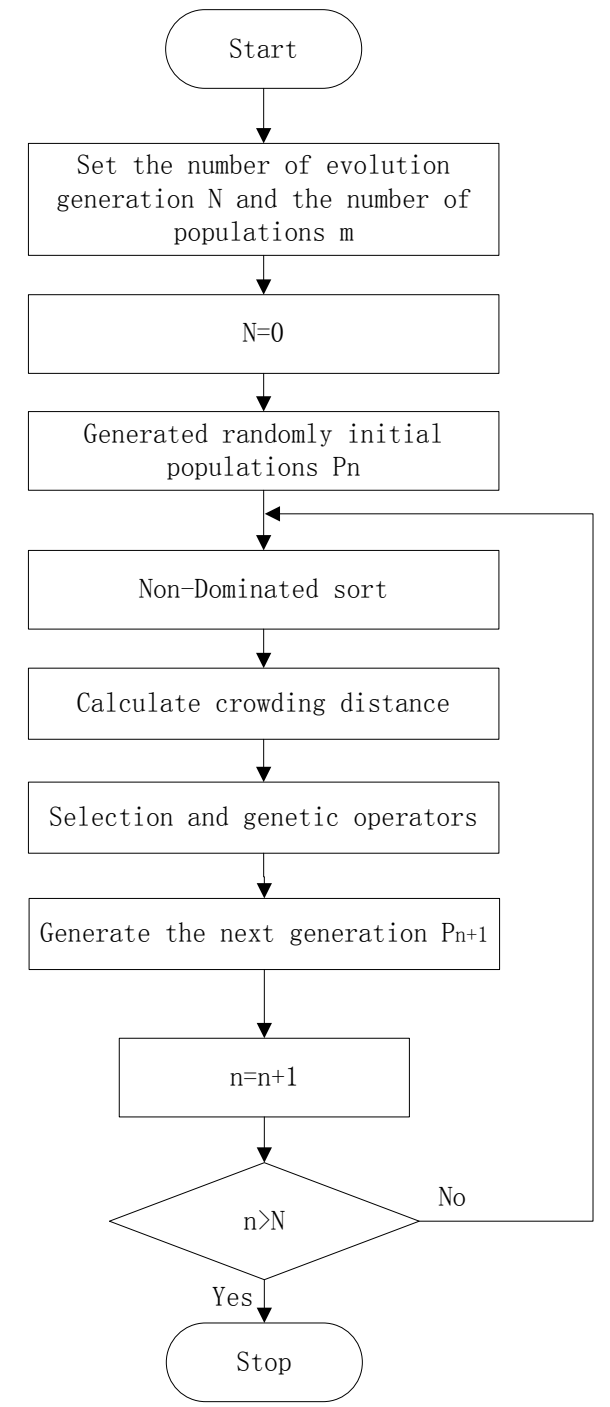

Fig. (4). NSGA-II algorithm proceduce.

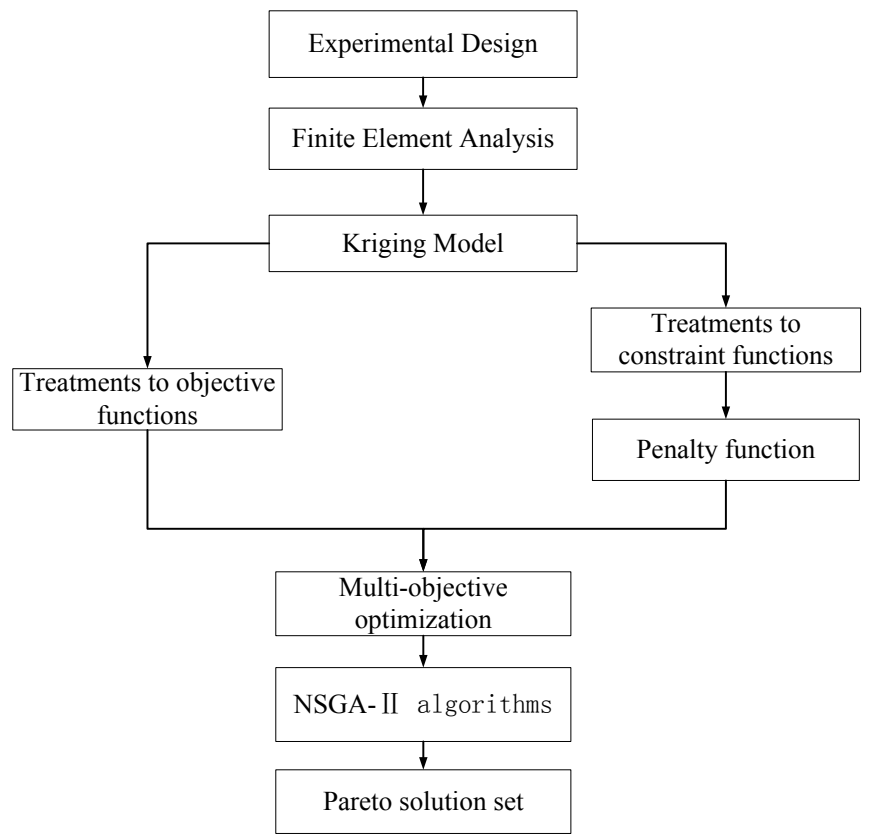

Fig. (5). The flow chart of crashworthiness optimal design for thinwalled rail.

\subsection{Experiment Design}

Experiment design-to seek for function relationship between design variables and optimization objectives, series of discrete sample points are supposed to be certain in given space at first. Latin square design, an efficient design procedure based on random sampling, can acquire larger information within limited number of times. 30 groups of experiment samples are gained by Latin square design in the passage. By finite element simulation, relevant total energy absorption, average impulsive load and maximum deformation are recorded of each thin-walled rail impact group.

\subsection{The Kriging Approximation Model}

As the highly nonlinear characteristics about the crash safety issues, it's difficult to find an implicit function expression between optimization objectives and design variables. The approximate model technology can predict response values of unknown points by utilizing known points to form fitting functions. The Kriging approximation model [5], a new approximate response surface methodology, exhibits stronger capability in predicting with two compartments - one parameter model and one nonparametric stochastic process. Here follows its expression:

$\tilde{y}(x)=f(x)+Z(x)$

Among the expression, $\tilde{y}(x)$ represents approximate function. $f(x)$ is polynomial regress function which is the global approximation of design space, while $Z(x)$ is a random process to approximate partial deviations. Usually take $N\left(0, \sigma^{2}\right)$ as normal distribution. In the sample space, variable have the same distribution, but not independence. The sample covariance of $x_{i}$ and $x_{j}$ can be expressed as:

$\operatorname{cov}\left[Z\left(x_{i}\right), Z\left(x_{j}\right)\right]=\sigma^{2} R\left(x_{i}, x_{j}\right)$

where $R\left(x_{i}, x_{j}\right)$ is the relevant function about the sample $x_{i}$ and $x_{j}$.

\subsection{Optimization Process}

Convert the target function and the constraint function to certainty function via uncertainty interval optimization algorithm, and then solve it by multi-objective optimization algorithm NSGA-II. In the optimization algorithm, population size is set to 100 , the number of offspring is set to 100 , the crossing-over rate is set to 0.9 and the aberration rate is set to 0.33 . Accuracy of Kriging approximation model is listed in Table 1, achieved by test data fitting. The values of $R^{2}$ are close to 1 , which means excellent accuracy of fitting functions. Fig. (6) is a Pareto set calculated, it can be seen from the figure that solution points are relatively concentrated. Total energy ranges from $10.58 \mathrm{KJ}$ to $29.15 \mathrm{KJ}$, whilst average impulsive load ranges from $9.49 \mathrm{KN}$ to $55.60 \mathrm{KN}$. Total energy absorption value increases with increasing average impulsive load. Eleven solutions listed are evenly chosen from Pareto set, as shown in Table 2. 
Table 1. Fitting accuracy of the approximate model.

\begin{tabular}{|c|c|}
\hline Kriging Model & Fit Factor $\boldsymbol{R}^{2}$ \\
\hline \hline Total energy absorption & 0.97 \\
\hline Average impulsive load & 0.97 \\
\hline Maximum deformation & 0.92 \\
\hline
\end{tabular}

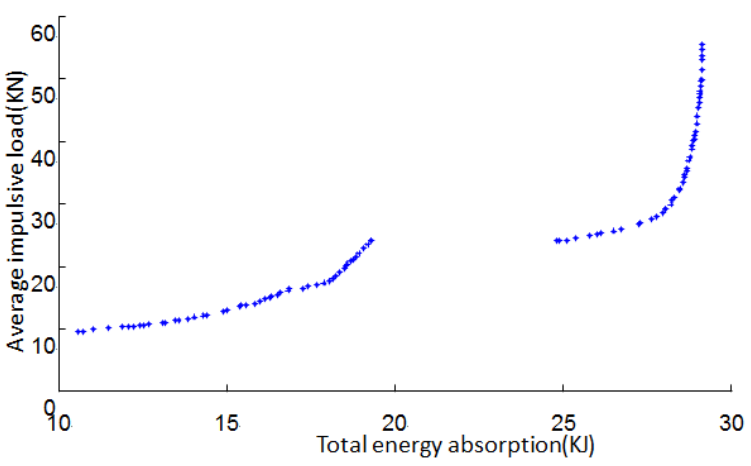

Fig. (6). Pareto optimal set achieved.

Table 3. Values of design variable to Pareto solution points.

\begin{tabular}{|c|c|c|c|c|c|}
\hline \multirow{2}{*}{ Pareto Solution Point } & \multicolumn{3}{|c|}{ Design Variables } & \multirow{2}{*}{ Total Energy Absorption (KJ) } & Average Impulsive Load (KN) \\
\cline { 2 - 5 } & $\mathbf{w}$ & $\mathbf{h}$ & $\mathbf{t}$ & 10.58 & 9.49 \\
\hline \hline 1 & 101.21 & 78.47 & 1.00 & 19.30 & 24.07 \\
\hline 50 & 97.67 & 120.0 & 1.22 & 29.15 & 55.60 \\
\hline 100 & 103.77 & 120.0 & 4.00 & & \\
\hline
\end{tabular}

Table 3 marks corresponding design variables of the $1^{\text {st }}$, $50^{\text {th }}$ and $100^{\text {th }}$ solution points in Pareto solution set. Referring to practical energy problems, pick the $100^{\text {th }}$ solution point in Pareto set as design variable to have greater amount of total energy absorption and pick the $1^{\text {st }}$ solution point in Pareto set as design variable to lower average compulsive load.

\section{CONCLUSION}

In this paper, the energy absorption characteristics of thin-walled rail under different collision boundary conditions have been optimized. An optimization method combined uncertainty interval algorithm, Kriging approximation model and NSGA-II genetic algorithm has been proposed. Select the structural parameters of thin-walled rail as research subjects, build collision model of crashworthiness by the Latin square design and explicit finite element method. Sled test is to verify the accuracy of finite element model. With fitting amongst total energy absorption, average impulsive load and maximum deformation achieved from finite element analysis, the Kriging approximation model was established. Likewise, the Pareto optimal solution set was resolved by leading uncertainty interval optimization and NSGA-II multi-objective genetic algorithm into thin-walled rail's crashworthiness optimization. In the aggregate, the optimized thin-walled rail structure is available to warrant excellent energy absorption attributes under various collision boundary conditions. The research methodology and conclusion in this paper offer possibility to design automobiles for all kinds of collision regulations.

Table 2. Eleven solutions evenly chosen from Pareto set.

\begin{tabular}{|c|c|c|}
\hline $\begin{array}{c}\text { Pareto Solution } \\
\text { Point }\end{array}$ & $\begin{array}{c}\text { Total Energy } \\
\text { Absorption (KJ) }\end{array}$ & $\begin{array}{c}\text { Average Impulsive } \\
\text { Load (KN) }\end{array}$ \\
\hline \hline 1 & 10.58 & 9.49 \\
\hline 10 & 12.69 & 10.63 \\
\hline 20 & 15.04 & 12.97 \\
\hline 30 & 16.57 & 15.74 \\
\hline 40 & 18.35 & 18.96 \\
\hline 50 & 19.30 & 24.07 \\
\hline 60 & 26.75 & 25.96 \\
\hline 70 & 28.31 & 30.97 \\
\hline 80 & 28.82 & 38.84 \\
\hline 90 & 29.07 & 47.04 \\
\hline 100 & 29.15 & 55.60 \\
\hline
\end{tabular}

\section{CONFLICT OF INTEREST}

The author confirms that this article content has no conflict of interest.

\section{ACKNOWLEDGEMENTS}

This work was financially supported by the National Natural Science Foundation of China (No. 51405035), Hunan Provincial Natural Science Foundation of China (No. 2015JJ6003), the open foundation of State Key Laboratory of Advanced Design and Manufacturing for Vehicle Body (No. 31375006) and the open foundation of Hunan Province Key Laboratory of Lightweight and Reliability Technology for Engineering Vehicle. This study and paper would not have been possible without their support.

\section{REFERENCES}

[1] S. N. Mohamed, N. K.Gupta, "Optimization of thin conical frusta for impact energy absorption", Thin-Walled Structures, vol. 46, pp. 653-666, 2008

[2] Y. Zhang, G.Y. Li, Z.H. Zhong, "Application of thin-walled beam lightweight based on reliability of multidisciplinary design optimization", China Mechanical Engineering, vol. 20, no. 15, pp. 1885-1889, 2009 
[3] C. Jiang, X. Han, G. R. Liu, "A nonlinear interval number programming method for uncertain optimization problems", European Journal of Operational Research, vol. 188, no. 1, pp. 1-13, 2008

[4] X.M. Dong, F.Y. Ding, Z. Guan, "Multi-objective optimization and performance research of magneto-rheological absorber under high speed". Journal of Mechanical Engineering, vol. 1, no. 5, pp. 127134,2014
[5] H. He, G. Zhu, C. He, "Crashworthiness Optimization Based on Kriging Metamodeling", Journal of Nanjing University of Aeronautics \& Astronautics, vol. 1, no. 5, pp. 297-303, 2014

Received: February 17, 2014

Revised: March 21, 2015

Accepted: June 9, 2015

(C) Gong et al.; Licensee Bentham Open.

This is an open access article licensed under the terms of the (https://creativecommons.org/licenses/by/4.0/legalcode ), which permits unrestricted, non-commercial use, distribution and reproduction in any medium, provided the work is properly cited. 